\title{
Elastic scattering of electromagnetic neutrinos on electrons
}

\section{Konstantin Kouzakov*}

Department of Nuclear Physics and Quantum Theory of Collisions, Faculty of Physics, Lomonosov Moscow State University, Moscow 119991, Russia

E-mail: kouzakovegmail.com

\section{Alexander Studenikin}

Department of Theoretical Physics, Faculty of Physics, Lomonosov Moscow State University, Moscow 119991, Russia

Joint Institute for Nuclear Research, Dubna 141980, Moscow Region, Russia

E-mail: studenik@srd.sinp.msu.ru

The electron angular distribution in the process of low-energy elastic neutrino-electron scattering is discussed in the context of neutrino electromagnetic properties. The formulas for the angular differential cross sections due to the electroweak and magnetic scattering channels are presented in the case of the neutrino collision with a free electron. Basic differences between the electroweak and magnetic contributions to the electron angular distribution are pointed out.

The European Physical Society Conference on High Energy Physics

5-12 July, 2017

Venice

${ }^{*}$ Speaker. 


\section{Introduction}

The progress of our knowledge about neutrino masses and mixing [1-3] makes it timely to explore neutrino properties and interactions beyond the standard model. A nonzero charge radius of the neutrino appears already in the standard model [4], and in the minimal extension of the standard model with right-handed neutrinos one obtains, in addition, nonzero values for neutrino magnetic and electric moments [5]. The manifestations of neutrino electromagnetic interactions [6-8] are searched in astrophysical environments, where neutrinos propagate over long distances in strong magnetic fields and dense matter [9], and in laboratory measurements of neutrinos from various sources. In the latter case, a very sensitive and widely used method is provided by the direct measurement of low-energy elastic (anti)neutrino-electron scattering in reactor, accelerator, and solar experiments. A typical strategy of such experiments consists in determining deviations of the energy spectrum of recoil electrons from the prediction of the standard model of the electroweak interaction. At the same time, an alternative way consists in studying the angle distribution of recoil electrons rather than their energy spectrum.

Below we present general formulas for the cross section, which is differential with respect to the electron solid angle. Then, we discuss specific features of the angular distributions of recoil electrons associated with the electroweak and magnetic scattering channels.

\section{The angular differential cross section}

We consider the process where an ultrarelativistic Dirac neutrino with flavor $\ell$ and energy $E_{v}$ originates from a source and elastically scatters on an electron in a detector at energy transfer $T$. The simplest model of the electron system in the detector is a free-electron model, where it is assumed that electrons are free and at rest. This approximation is supposed to be generally applicable if the energy-transfer value $T$ is much larger than the electron binding energy in the detector.

In the scattering experiments the observables are the kinetic energy $T_{e}$ of the recoil electron and/or its solid angle $\Omega_{e}$. From the energy-momentum conservation one gets

$$
T_{e}=T, \quad \cos \theta_{e}=(1+\xi) \sqrt{\frac{T}{T+2 m_{e}}},
$$

where $\theta_{e}$ is the angle of the recoil electron with respect to the neutrino beam and $\xi=m_{e} / E_{v}$. The angular differential cross section, which can be readily derived from the energy differential cross section [10], is given by

$$
\frac{d \sigma}{d \Omega_{e}}=\frac{d \sigma_{(w)}}{d \Omega_{e}}+\frac{d \sigma_{(\mu)}}{d \Omega_{e}}
$$

where

$$
\begin{aligned}
\frac{d \sigma_{(w)}}{d \Omega_{e}}= & \frac{G_{F}^{2} m_{e}^{2}}{\pi^{2}} \frac{(1+\xi)^{2} \cos \theta_{e}}{\left[(2+\xi) \xi+\sin ^{2} \theta_{e}\right]^{2}}\left\{A_{\ell}+B_{\ell}+2 C_{\ell}\right. \\
& \left.+\left(A_{\ell}+B_{\ell}-2 C_{\ell}\right)\left[\frac{\xi^{2}+(1+2 \xi) \sin ^{2} \theta_{e}}{(2+\xi) \xi+\sin ^{2} \theta_{e}}\right]^{2}+\frac{2\left(B_{\ell}-A_{\ell}\right) \xi^{2} \cos ^{2} \theta_{e}}{(2+\xi) \xi+\sin ^{2} \theta_{e}}\right\}
\end{aligned}
$$


is the electroweak contribution, and

$$
\frac{d \sigma_{(\mu)}}{d \Omega_{e}}=\frac{\alpha^{2}}{m_{e}^{2}}\left|\mu_{\ell}^{e f f}\right|^{2} \frac{(1+\xi)^{2}\left[\xi^{2}+(1+2 \xi) \sin ^{2} \theta_{e}\right]}{\cos \theta_{e}\left[(2+\xi) \xi+\sin ^{2} \theta_{e}\right]^{2}}
$$

is the magnetic contribution. In the case of short-baseline scattering experiments, one has (hereafter we assume that neutrinos are electrically neutral)

$$
\begin{aligned}
& A_{\ell}=\left(g_{V}+\delta_{\ell e}+\eta\left\langle r_{v}^{2}\right\rangle_{\ell \ell}\right)^{2}+\eta^{2} \sum_{\ell^{\prime}=e, \mu, \tau}\left(1-\delta_{\ell^{\prime} \ell}\right)\left|\left\langle r_{v}^{2}\right\rangle_{\ell^{\prime} \ell}\right|^{2}, \quad B_{\ell}=\left(g_{A}+\delta_{\ell e}\right)^{2}, \\
& C_{\ell}=\left(g_{V}+\delta_{\ell e}\right)\left(g_{A}+\delta_{\ell e}\right)+\left(g_{A}+\delta_{\ell e}\right) \eta\left\langle r_{v}^{2}\right\rangle_{\ell \ell}, \quad\left|\mu_{\ell}^{e f f}\right|^{2}=\sum_{\ell^{\prime}=e, \mu, \tau}\left|\left(\mu_{v}\right)_{\ell^{\prime} \ell}\right|^{2},
\end{aligned}
$$

with $g_{V}=2 \sin ^{2} \theta_{W}-1 / 2, g_{A}=-1 / 2, \eta=\frac{2}{3} m_{W}^{2} \sin ^{2} \theta_{W}$. The quantities

$$
\left\langle r_{v}^{2}\right\rangle_{\ell^{\prime} \ell}=\sum_{j, k=1}^{3} U_{\ell k}^{*} U_{\ell^{\prime} j}\left\langle r_{v}^{2}\right\rangle_{j k} \quad \text { and } \quad\left(\mu_{v}\right)_{\ell^{\prime} \ell}=\sum_{j, k=1}^{3} U_{\ell k}^{*} U_{\ell^{\prime} j}\left(\mu_{v}\right)_{j k}
$$

are the neutrino charge radius and magnetic moment (measured in units of $\mu_{B}$ ) of the diagonal $\left(\ell=\ell^{\prime}\right)$ or transition $\left(\ell \neq \ell^{\prime}\right)$ type in the flavor basis, which include also the effects of the neutrino anapole and electric moments, respectively.

\section{Properties of the electron angular distribution}

The angular distribution of recoil electrons is confined within the forward hemisphere, i.e. $0 \leq \theta_{e} \leq \pi / 2$. Let us first discuss the $\theta_{e}=0$ case, that is, when the kinetic energy of the recoil electron takes on the maximal kinematically allowed value $T_{e}^{\max }=E_{v} /(1+\xi / 2)$. The electroweak and magnetic components of the angular differential cross section (2.2) in this case are given by

$$
\begin{aligned}
& \frac{d \sigma_{(w)}}{d \Omega_{e}}\left(\theta_{e}=0\right)=\frac{4 G_{F}^{2} E_{v}^{2}}{\pi^{2}} \frac{(1+\xi)^{2}}{(2+\xi)^{4}}\left[A_{\ell}+B_{\ell}(1+\xi)^{2}+2 C_{\ell}(1+\xi)\right], \\
& \frac{d \sigma_{(\mu)}}{d \Omega_{e}}\left(\theta_{e}=0\right)=\frac{\alpha^{2}}{m_{e}^{2}}\left|\mu_{\ell}^{e f f}\right|^{2} \frac{(1+\xi)^{2}}{(2+\xi)^{2}} .
\end{aligned}
$$

It can be seen that the relative contribution of the magnetic scattering channel to the electron angular distribution in the forward direction grows with decreasing the neutrino energy value.

When $\theta_{e} \rightarrow \pi / 2$, the electron kinetic energy is $T_{e} \rightarrow 0$. The electroweak and magnetic angular differential cross sections in the vicinity of $\theta_{e}=\pi / 2$ behave as follows:

$$
\begin{aligned}
& \frac{d \sigma_{(w)}}{d \Omega_{e}}\left(\theta_{e} \rightarrow \pi / 2\right)=\frac{2 G_{F}^{2} m_{e}^{2}}{\pi^{2}} \frac{A_{\ell}+B_{\ell}}{(1+\xi)^{2}} \cos \theta_{e}, \\
& \frac{d \sigma_{(\mu)}}{d \Omega_{e}}\left(\theta_{e} \rightarrow \pi / 2\right)=\frac{\alpha^{2}}{m_{e}^{2}}\left|\mu_{\ell}^{e f f}\right|^{2} \frac{1}{\cos \theta_{e}} .
\end{aligned}
$$

The same as in the $\theta_{e}=0$ case, the relative contribution of the magnetic scattering channel in the limit $\theta_{e} \rightarrow \pi / 2$ grows with decreasing the neutrino energy value. The electroweak and magnetic components of the electron angular distribution are markedly different in the vicinity of $\pi / 2$, namely the former tends to zero as $\propto \cos \theta_{e}$ while the latter tends to infinity as $\propto 1 / \cos \theta_{e}$. This means that the closer the electron angle to $\pi / 2$ the higher sensitivity to neutrino magnetic moments one might expect when measuring the electron angular distribution. 


\section{Concluding remarks}

We have outlined some basic features of the electron angular distribution in the low-energy elastic neutrino-electron scattering under the assumption that the free-electron model is applicable. However, when the energy-transfer value $T$ is comparable to the electron binding energy in the detector, this model becomes not generally valid anymore. The role of the electron-binding effects in the neutrino scattering on atomic electrons is well studied in the case of the cross section differential in $T$, i.e. $d \sigma / d T$. This cross section to a good approximation equals to the free-electron one above the ionization threshold and vanishes otherwise (see, for instance, Ref. [11] and references therein). The impact of electron-binding effects on the angular differential cross section $d \sigma / d \Omega_{e}$ is, in general, more involved. In particular, after being knocked by the neutrino the atomic electron can recoil from the atomic nucleus. This leads to a nonzero electron angular distribution in the backward hemisphere, what is kinematically forbidden in the free-electron model.

\section{Acknowledgements}

This work was supported by the Russian Foundation for Basic Research under grants No. 1602-01023 A and No. 17-52-53133 GFEN_a.

\section{References}

[1] S. Bilenky, Introduction to the physics of massive and mixed neutrinos. Springer, 2010.

[2] Z.-z. Xing and S. Zhou, Neutrinos in particle physics, astronomy and cosmology. Zhejiang University Press, 2011.

[3] S. F. King, Models of neutrino mass, mixing and CP violation, J. Phys. G: Nucl. Part. Phys. 42 (2015) 123001, [hep-ph/1510.02091].

[4] J. Bernabeu, L. G. Cabral-Rosetti, J. Papavassiliou and J. Vidal, On the charge radius of the neutrino, Phys. Rev. D 62 (2000) 113012, [hep-ph/ 0008114 ].

[5] K. Fujikawa and R. Shrock, The Magnetic Moment of a Massive Neutrino and Neutrino Spin Rotation, Phys. Rev. Lett. 45 (1980) 963.

[6] C. Broggini, C. Giunti and A. Studenikin, Electromagnetic Properties of Neutrinos, Adv. High Energy Phys. 2012 (2012) 459526, [hep-ph/1207. 3980].

[7] C. Giunti and A. Studenikin, Neutrino electromagnetic interactions: A window to new physics, Rev. Mod. Phys. 87 (2015) 531-591, [hep-ph/1403.6344].

[8] C. Giunti, K. A. Kouzakov, Y.-F. Li, A. V. Lokhov, A. I. Studenikin and S. Zhou, Electromagnetic neutrinos in laboratory experiments and astrophysics, Ann. Phys. (Berlin) 528 (2016) 198-215, [hep-ph/1506.05387].

[9] G. Raffelt, Stars as laboratories for fundamental physics: The astrophysics of neutrinos, axions, and other weakly interacting particles. University of Chicago Press, 1996.

[10] K. A. Kouzakov and A. I. Studenikin, Electromagnetic properties of massive neutrinos in low-energy elastic neutrino-electron scattering, Phys. Rev. D 95 (2017) 055013, [hep-ph/1703.00401].

[11] K. A. Kouzakov and A. I. Studenikin, Theory of neutrino-atom collisions: the history, present status and BSM physics, Adv. High Energy Phys. 2014 (2014) 569409, [hep-ph/1406.4999]. 